\title{
BMJ Open Safety and effectiveness of dose-sparing strategies for intramuscular seasonal influenza vaccine: a rapid scoping review
}

\author{
Carole Lunny (D) , ${ }^{1,2}$ Jesmin Antony, ${ }^{3}$ Patricia Rios, ${ }^{4}$ Chantal Williams, ${ }^{4}$ \\ Naveeta Ramkissoon, ${ }^{2}$ Sharon E Straus, ${ }^{3,5}$ Andrea C Tricco (1) ${ }^{2}$
}

To cite: Lunny C, Antony J, Rios P, et al. Safety and effectiveness of dose-sparing strategies for intramuscular seasonal influenza vaccine: a rapid scoping review. BMJ Open 2021;11:e050596. doi:10.1136/ bmjopen-2021-050596

- Prepublication history and additional supplemental material for this paper are available online. To view these files, please visit the journal online (http://dx.doi.org/10.1136/ bmjopen-2021-050596).

Received 25 February 2021 Accepted 31 August 2021

\section{Check for updates}

(c) Author(s) (or their employer(s)) 2021. Re-use permitted under CC BY-NC. No commercial re-use. See rights and permissions. Published by BMJ.

${ }^{1}$ Cochrane Hypertension Review Group and the Therapeutics Initiative, UBC, Vancouver, British Columbia, Canada

${ }^{2}$ Knowledge Translation Program, St Michael's Hospital, Li Ka Shing Knowledge Institute, Toronto, Ontario, Canada

${ }^{3}$ Li Ka Shing Knowledge Institute, St Michael's Hospital, Toronto, Ontario, Canada ${ }^{4}$ Independent Researcher, Toronto, Ontario, Canada

${ }^{5}$ Geriatric Medicine, University of Toronto, Toronto, Ontario, Canada

Correspondence to

Dr Carole Lunny;

carole.lunny@ubc.ca

\section{ABSTRACT}

Background The objective of this rapid scoping review was to identify studies of dose-sparing strategies for administration of intramuscular seasonal influenza vaccines in healthy individuals of all ages.

Methods Comprehensive literature searches were executed in MEDLINE, Embase and the Cochrane library. The grey literature was searched via international clinical trial registries for relevant studies published in English in the last 20 years. We included studies in healthy humans of any age that used any dose-sparing strategy to administer intramuscular seasonal influenza vaccines. Title/abstract and full-text screening were carried out by pairs of reviewers independently. Data extraction was conducted by a single reviewer and verified by a second reviewer. Our outcomes were influenza infections, intensive care unit admission, pneumonia, hospitalisations, adverse events and mortality. Results were summarised descriptively.

Results A total of 13 studies with 10351 participants were included in the review and all studies were randomised controlled trials (RCTs) conducted between 2006 and 2019. The most common interventions were the trivalent influenza vaccine $(n=10)$, followed by the quadrivalent influenza vaccine $(n=4)$. Nine studies included infants/toddlers 6-36 months old and one of these studies also included children and adolescents. In these nine studies, no clinical effectiveness outcomes were reported. Of the four adult studies ( $\geq 18$ years), two studies reported on effectiveness outcomes, however, only one RCT reported on laboratory-confirmed influenza.

Conclusions Due to the low number of studies in healthy adults and the lack of studies assessing confirmed influenza and influenza-like illness, there remains a need for further evaluation.

\section{BACKGROUND}

The symptoms of novel COVID-19 closely mimic those of seasonal influenza vaccine and health officials recommend vaccination against the influenza to limit confounding of influenza symptoms with COVID-19 symptoms. An anticipated shortage in influenza vaccine supplies was of concern. ${ }^{1}$ This anticipated
Strengths and limitations of this study

- This rapid scoping review was conducted within a 6 -week timeline and the methods were tailored to provide results to the stakeholders within 4 weeks.

- We did not restrict the search dates and study screening was completed in independently by two reviewers.

- We limited the selection of studies to those published in the English language, and data extraction was conducted by one abstractor and one verifier.

- Twelve dose-sparing randomised control trials were not included in the review because they did not include vaccine interventions that were deemed of interest to the stakeholders and/or did not provide sufficient data.

shortage did not happen, however, and in the 2019-2020 influenza season, influenza vaccination coverage among adults (42\%) was similar to the previous season $(42 \%)$. This question of vaccine shortage remains relevant in Canada and other jurisdictions for future COVID-19 and flue seasons. As a potential solution, health officials were interested in assessing the effectiveness of fractional dosing (eg, half-doses) of currently available intramuscular (IM) influenza vaccines.

Fractional dosing, or dose-sparing, strategies are those where less than the standard dose of haemagglutinin (HA) antigen, and thus less volume of vaccine, is administered, increasing the overall number of influenza vaccine doses available. In Canada, influenza vaccines are currently authorised for IM administration only, apart from the live-attenuated influenza vaccine, which is administered intranasally. ${ }^{2}$ Standard dose influenza vaccines contain $15 \mu \mathrm{g}$ of HA per strain and are delivered in $0.5 \mathrm{~mL}$ volume. Therefore, the total amount of $\mathrm{HA}$ in 
standard dose trivalent vaccines is $45 \mu \mathrm{g}$, and the total amount of $\mathrm{HA}$ in standard dose quadrivalent vaccines is $60 \mu \mathrm{g}$.

A scoping review of all the available dose-sparing strategies for IM administration of seasonal influenza vaccines currently approved in Canada for healthy populations had not been systematically conducted. With the resource constraints for the influenza season due to COVID-19, there was a need to scope the evidence on the safety and effectiveness of dose-sparing strategies for IM administration of seasonal influenza vaccines. The objective of this rapid scoping review was to identify studies of dosesparing strategies for administration of IM seasonal influenza vaccines in healthy individuals of all ages. The results of this scoping review were used to inform a systematic review with meta-analysis by National Advisory Committee on Immunization (NACI) on the same topic. ${ }^{3}$

\section{METHODS}

The Centre for Immunisation and Respiratory Infectious Diseases of the Public Health Agency of Canada (PHAC) commissioned a rapid scoping review on the available methods for fractional dosing of seasonal influenza vaccines through the Canadian Institutes of Health Research Drug Safety and Effectiveness Network with a 6-week timeline for preliminary results.

\section{Protocol}

The methods for this review were guided by the updated reviewer manual for scoping reviews published by JBI (https://jbi.global/) and the WHO's guide to rapid reviews. ${ }^{45}$ Results are reported according to the Preferred Reporting Items for Systematic Reviews and Meta-analyses extension to Scoping Reviews. ${ }^{6}$ A protocol for this rapid scoping review was disseminated through the Open Science Framework registry (https://osf.io/8mwz2/).

\section{Patient and public involvement statement}

No patients or the public were involved in this rapid scoping review.

\section{Literature search}

Comprehensive literature searches were developed and executed by an experienced librarian in Ovid MEDLINE (online supplemental appendix 1, EMBASE using the OVID interface (online supplemental appendix 2), and the Cochrane library between 1946 and May 2020 (online supplemental appendix 3). The literature search was peer reviewed by a second librarian using the PRESS checklist (https://www.cadth.ca/resources/finding-evidence/ press). The grey (ie, difficult to locate or unpublished) literature was searched via international clinical trial registries (ie, clinicaltrials.gov, European Union clinical trial register). References of relevant systematic reviews and included studies were also scanned.

\section{Eligibility criteria}

The eligibility criteria followed the Population, Intervention, Comparators, Outcome, Study design (PICOS) framework as follows:

- Population: Healthy humans of any age. Immunocompromised populations and animal studies were excluded. Examples of persons with weakened immune systems include those with HIV/AIDS; cancer and transplant patients who are taking certain immunosuppressive drugs; and those with inherited diseases that affect the immune system (eg, congenital agammaglobulinaemia, congenital IgA deficiency). ${ }^{7}$

- Intervention: Any dose-sparing strategy used to administer IM seasonal influenza vaccines (eligible vaccines listed in online supplemental appendix 4). Eligible strategies included, but were not limited to, administrating less than the standard 15 ug HA antigen using multidose vials (MDV), half dosing or preformulated products with reduced antigen quantity, or with revised vaccine dose schedules. Any studies examining monovalent pandemic vaccines, specialty/experimental vaccines (eg, high dose), whole virus vaccines or other routes of administration (eg, intranasal, intradermal (ID)) were not eligible. Only vaccine products approved for use in Canada or equivalent formulations approved for use in other countries were eligible for inclusion. Concomitant administration with other vaccine products were included only if administered to both the intervention and the comparator groups.

- Comparator: Any of the interventions listed above, no intervention or placebo.

- Outcomes: Lboratory-confirmed influenza infection (primary outcome), influenza-like illness or clinical/ symptomatic diagnosis of influenza, hospitalisation, intensive care unit (ICU) admission, pneumonia, mortality and adverse events (local/systemic reactogenicity, vascular-related, serious). Reactogenicity represents the physical manifestation of the inflammatory response to vaccination, and can include injection-site pain, redness, swelling or induration at the injection site, as well as systemic symptoms, such as fever, myalgia or headache. ${ }^{8}$ Immunogenicity outcomes were not abstracted, but these studies were flagged for NACI.

- Study designs: Randomised controlled trials (RCTs), non-randomised studies (eg, quasi-RCTs, nonrandomised trials, interrupted time series, controlled before after) and observational studies (eg, cohort, case control) were included. Studies must have had a control or comparator group in order to be eligible for inclusion and as such, cross-sectional, case series, case reports and qualitative studies were excluded.

- Publication status: We included full text and abstracts if they included data on safety or effectiveness.

Inclusion was also limited to studies written in the English language due to the short timelines for the conduct of this review. 


\section{Study selection}

A screening form based on the eligibility criteria was prepared and pilot-tested with 30 studies with all members of the review team until sufficient agreement $(>75 \%)$ was reached prior to both title/abstract (level 1) and full-text (level 2) screening. Subsequent screening at level 1 and level 2 was completed by two reviewers working independently using the Knowledge Translation Programme's proprietary screening software (synthesi. SR) ${ }^{9}$ Any discrepancies between reviewers were consistently resolved by a third independent reviewer.

\section{Data extraction}

Items for data collection included study characteristics (study design, year of publication, country of conduct, multicentre vs single site), patient characteristics (mean age, age range, sex, vaccination history), intervention details (type of vaccine, vaccine manufacturer, dose, timing and administration of treatment), comparator details (comparator intervention, dose) and outcome results (influenza infections, ICU admission, pneumonia, hospitalisations, adverse events, mortality) at the longest duration of follow-up.

A standardised form for data extraction was developed and pilot tested by the entire review team using two preselected full-text RCTs to ensure understanding of the data items to be extracted, and congruence among reviewers. All included studies were extracted by one reviewer independently and then verified by a second reviewer.

\section{Risk of bias assessment}

As this was a scoping review, the risk of bias of studies was not assessed. ${ }^{4}$

\section{Synthesis}

The synthesis involved providing a descriptive summary of included studies with summary tables and detailed tables of study results. Study results were organised and tabulated according to patients (children vs adults), interventions and outcomes and where available information on relevant subgroups.

\section{RESULTS}

\section{Literature search}

We screened 2378 titles and abstracts from our database search and an additional 13 citations located through searching the grey literature and scanning references. Of these, 144 potentially relevant full-text articles were screened for eligibility (figure 1). Twelve studies that assessed dose-sparing strategies were excluded during full-text screening because the vaccine under study was not of interest or unclearly reported. We contacted authors of these 12 unclear studies and received 1 response confirming the vaccine was not of interest (see list of excluded studies in online supplemental appendix 5). Subsequently, 13 RCTs were included; 5 trial protocols were found and were denoted as duplicate/companion reports. No non-randomised or observational studies were found that fulfilled the eligibility criteria.

\section{Study characteristics}

Table 1 summarises the characteristics of the 13 RCTs published between 2006 and 2019 and and conducted mainly in the USA, followed by Mexico, Canada and Finland. The majority of the studies evaluated trivalent vaccines $(10 / 13(77 \%))$ and most were conducted in the 6-36 months old paediatric population $(9 / 13(69 \%))$. Almost all studies reported on reactogenicity and/or other adverse events, but only two studies reported on the effectiveness of our outcomes of interest (ie, laboratoryconfirmed influenza and influenza-like illness).

Full study and patient characteristic details for each study are reported in online supplemental appendix 6 and treatment and outcome details in online supplemental appendix 7 .

\section{RCTs in healthy children ( $<18$ years old)}

Nine studies included infants/toddlers 6-36 months old and one study also included children and adolescents (table 2). None of these studies reported results on the effectiveness outcomes that were relevant to our review and established a priori, however, all of them reported on safety outcomes.

\section{Safety outcomes \\ Trivalent influenza vaccines}

Six of the included RCTs assessed trivalent influenza vaccines (TIV) in young children (6-36 months) and reported on local and systemic reactogenicity outcomes and other adverse events. ${ }^{10-15}$ Two RCTs compared the administration of full $(0.5 \mathrm{~mL})$ and half $(0.25 \mathrm{~mL})$ doses of the same standard $15 \mu \mathrm{g} /$ strain vaccine. ${ }^{11}{ }^{15}$ The first RCT compared two full vs two half doses of TIV in previously unimmunised infants (6-11 months) and toddlers (12-23 months) using Vaxigrip (15 $\mu \mathrm{g} / \mathrm{strain}) .{ }^{11}$ The study found that in the infants group, two full $0.5 \mathrm{~mL}$ doses of vaccine did not increase reactogenicity. Local reactions were less common in infants than toddlers and more common with full doses versus half doses, but the differences were not statistically significant. An identified clinical trial registry compared a single IM injection of $0.5-0.25 \mathrm{~mL}$ of FLUAD or Agrippal and showed comparable numbers of children with reactogenicity outcomes and other adverse events across the groups, but no significance levels or conclusions were provided by the investigators on contact. ${ }^{15}$

The objective of three of the included RCTs was to examine the impact of administering the full adult dose of $15 \mu \mathrm{g} /$ strain vaccines compared with the usual children's dose of $7.5 \mu \mathrm{g} /$ strain in infants and toddlers. ${ }^{12-14}$ A multicentre RCT was conducted in Canada assessing the safety of full-dose Fluviral TIV $(15 \mu \mathrm{g} / \mathrm{strain})$ compared with the half-dose $(7.5 \mu \mathrm{g} /$ strain) and an active comparator Vaxigrip ( $7.5 \mu \mathrm{g} /$ strain).$^{12}$ Compared with the half-dose, the full-dose vaccine resulted in clinically similar reactogenicity 
and safety. A similar three-arm RCT to assess the use of Fluarix at two different dose levels $(7.5 \mu \mathrm{g} / \mathrm{strain}$ and $15 \mu \mathrm{g} /$ strain) compared with an established control vaccine Fluzone $(7.5 \mu \mathrm{g} / \mathrm{strain})$ also found the reactogenicity and safety profile of Fluarix did not appear to be affected by doubling the dose, but one participant in the $15 \mu \mathrm{g}$ group had two serious adverse events (apnea and cyanosis) that were considered by the investigator to be possibly related to vaccination. ${ }^{13}$ A third multicentre RCT compared the $15 \mu \mathrm{g} /$ strain formulation to the $7.5 \mu \mathrm{g} / \mathrm{strain}$ formulation of Fluzone (Sanofi Pasteur) administered to young children across multiple influenza seasons. ${ }^{14}$ This study also found no statistically significant differences between the full-dose or half-dose groups for systemic reactions, local reactions or adverse events when both seasons were combined; however, in the 2011-2012 season, 8 of $48(16.7 \%)$ participants in the half-dose group compared with 32 of $96(33.3 \%)$ in the full-dose group had increased redness at the injection site $(\mathrm{p}<0.05)$.

Della Cioppa et al was the only trial that compared the safety and tolerability of both TIV and quadrivalent influenza vaccines (QIV) vaccine formulations. ${ }^{10}$ The vaccine arms of interest were a QIV $15 \mu \mathrm{g} / \mathrm{strain}$, TIV $15 \mu \mathrm{g}$ / strain, QIV $7.5 \mu \mathrm{g} / \mathrm{strain}$, TIV $7.5 \mu \mathrm{g} / \mathrm{strain}$ and a control Vaxigrip TIV $7.5 \mu \mathrm{g} /$ strain vaccine. Reactogenicity of the $7.5 \mu \mathrm{g}$ TIV/QIV formulations was slightly lower than for the corresponding $15 \mu \mathrm{g}$ formulations, but there was no difference in reactogenicity between TIV and QIV vaccines.

\section{Quadrivalent influenza vaccines}

Four of the included RCTs evaluated QIV in children. ${ }^{10-16}$ All of the studies reported reactogenicity outcomes and other adverse events. Della Cioppa et al RCT reported both TIV and QIV vaccines and the results are reported above. ${ }^{10}$ Two studies compared
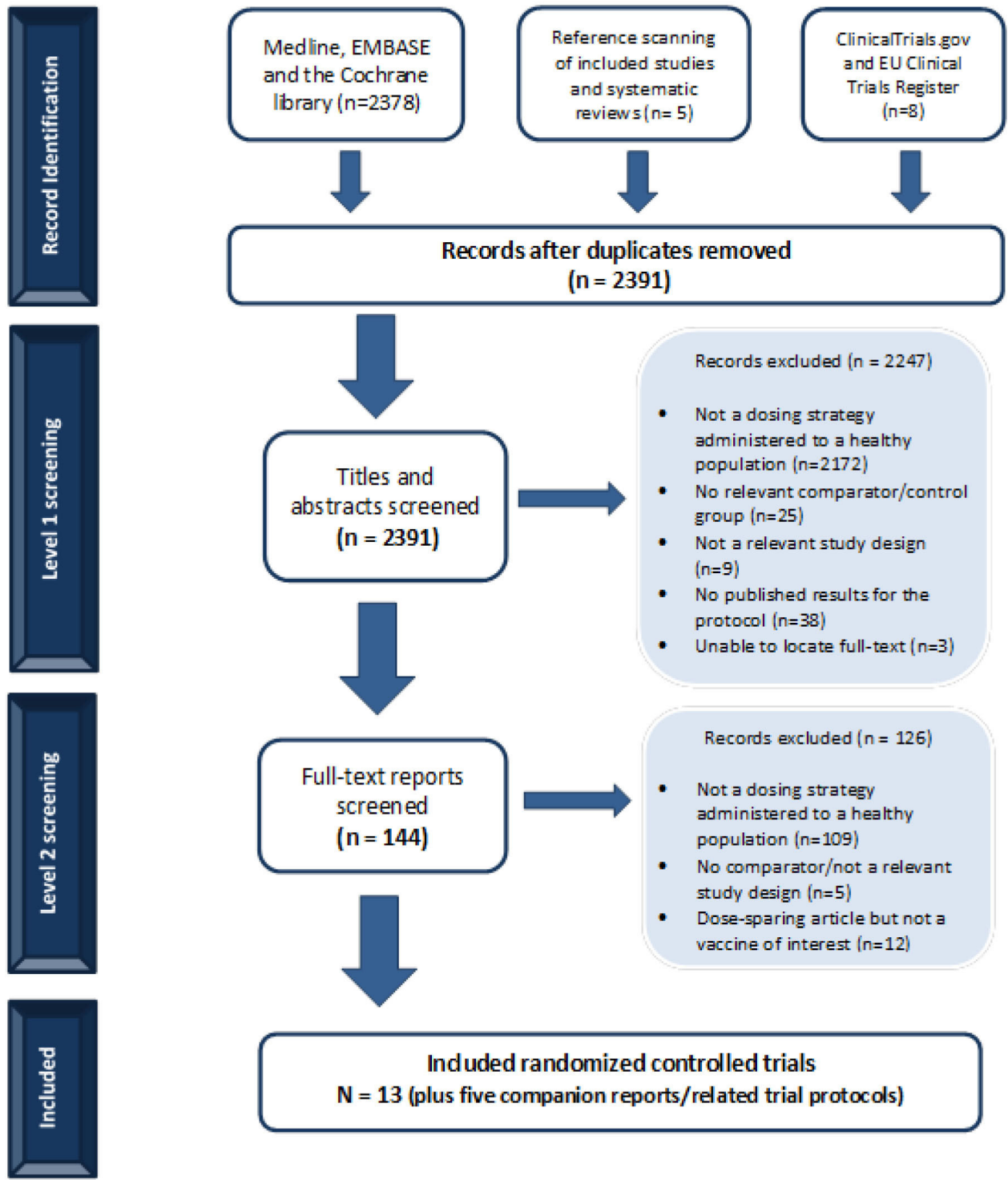

\section{Ind uded randomized controlled trials $\mathrm{N}=13$ (plus five companion reports/related trial protocols)}

Figure 1 Flow chart of studies included in the review. Study flow diagram. 
full-dose QIV to paediatric $7.5 \mu \mathrm{g} /$ strain Fluzone. In the first RCT, full dose Fluzone had a similar safety profile to half-dose Fluzone with a single adverse event being attributed to the study vaccine. ${ }^{18}$ Similarly, the second study found that full-dose Flulaval may improve protection against influenza in some young children when compared with low-dose Fluzone, and in this RCT, none of the adverse events were considered to be study related as reported by the investigator. ${ }^{16}$ The final trial evaluated Vaxigrip Tetra $(15 \mu \mathrm{g} / \mathrm{strain})$ administered to children and adolescents in two different formats. ${ }^{17}$ Vaxigrip administered as a single dose using a prefilled syringe (PFS) was compared with a 10-dose MDV. Systemic reactions were reported in more infants aged 6-35 months in the MDV group than in the PFS group; however, this difference was not clinically significant. The authors concluded that there was no difference in reactogenicity or safety between the two vaccine formats in infants, children and adolescents.

\section{RCTs in healthy adults ( $\geq 18$ years old)}

One RCT included healthy adults over 18 years, two studies included healthy adults from 18 to 45 and 18-65 years old, and one study included older healthy adults ( $\geq 65$ years) (table 3 ). Two studies reported on effectiveness outcomes and three on reactogenicity and other adverse events. All four RCTs evaluated Fluzone QIV.

\section{Effectiveness outcomes}

Two of the included RCTs that examined the same vaccine (Fluzone manufactured by Aventis Pasteur) in healthy adult populations reported effectiveness outcomes. Only one study by Kramer et al included lab-confirmed influenza infection, ${ }^{19}$ two reported influenza like illness, ${ }^{19} 20$ and one reported hospitalisations or emergency room visits after vaccination. ${ }^{20}$ The RCT by Kramer et al found that $3.6 \%$ of participants receiving a $15 \mu \mathrm{g} /$ strain dose of vaccine reported influenza like illness compared with $6.8 \%$ of participants that received a $7.5 \mu \mathrm{g} /$ strain dose. $^{19}$ However, only one participant that received the full dose $15 \mu \mathrm{g} / \mathrm{strain}$ was confirmed via laboratory analysis to have influenza, and no patients in the half-dose arm got laboratory confirmation. The authors concluded that halfdose and full-dose vaccinations appear to be similarly effective for influenza like illness and similar symptom surveys between both groups but acknowledge that further studies examining immunogenicity are needed to confirm.

A similar RCT by Engler et al that compared a $15 \mu \mathrm{g} /$ strain dose of Fluzone vaccine to a $7.5 \mu \mathrm{g} / \mathrm{strain}$ dose found equal proportions of participants reporting influenza like illness $(9.7 \%$ vs $9.9 \%)$ and hospitalisations or emergency room visits $(0.3 \%$ vs $0.2 \%) .^{20}$ The authors found the relative risk of medical visits or hospitalisations between both groups was the same even when adjusting
Table 1 Characteristics of included studies $(n=13)$

\begin{tabular}{|c|c|c|}
\hline Characteristics & Category & $\begin{array}{l}\text { Frequency } \\
(\%)\end{array}$ \\
\hline \multirow{3}{*}{$\begin{array}{l}\text { Date of } \\
\text { publication }\end{array}$} & 2006-2010 & $4(30.8)$ \\
\hline & 2011-2015 & $5(38.4)$ \\
\hline & $2016-2020$ & $4(30.8)$ \\
\hline \multirow{2}{*}{$\begin{array}{l}\text { Multicentre or } \\
\text { single site }\end{array}$} & Multicentre & $8(61.5)$ \\
\hline & Single centre & $2(15.4)$ \\
\hline \multirow{8}{*}{$\begin{array}{l}\text { Countries of } \\
\text { conduct }^{*}\end{array}$} & USA & $8(61.5)$ \\
\hline & Mexico & $3(23.1)$ \\
\hline & Canada & $2(15.4)$ \\
\hline & Finland & $2(15.4)$ \\
\hline & Belgium & $1(7.7)$ \\
\hline & Hong Kong & $1(7.7)$ \\
\hline & Taiwan & $1(7.7)$ \\
\hline & Thailand & $1(7.7)$ \\
\hline \multirow[t]{4}{*}{ Populations ${ }^{\star} \dagger$} & Infants/toddlers (6-36 months) & 9 (69.2) \\
\hline & Children (37 months - 17 years) & $1(7.7)$ \\
\hline & Adults (18-64 years) & $3(23.1)$ \\
\hline & Older adults $(\geq 65)$ & $1(7.7)$ \\
\hline \multirow[t]{2}{*}{ Treatments* $\ddagger$} & Trivalent influenza vaccine (TIV) & $10(76.9)$ \\
\hline & $\begin{array}{l}\text { Quadrivalent influenza vaccine } \\
\text { (QIV) }\end{array}$ & $4(30.8)$ \\
\hline \multirow[t]{3}{*}{ Outcomes* } & Effectiveness & $2(15.4)$ \\
\hline & $\begin{array}{l}\text { Local and systemic } \\
\text { reactogenicity }\end{array}$ & 12 (92.3) \\
\hline & Adverse events & $10(76.9)$ \\
\hline
\end{tabular}

*Each study can fit into more than one category so the total percentage will not add up to $100 \%$.

†One study includes both infants/toddlers and children, and another includes both adults and seniors.

‡One study includes both TIV and QIV arms.

for age and that age, sex, nor dose had an influence on the severity of influenza like illness symptoms.

\section{Safety outcomes}

Three of the included studies in adult populations reported adverse events that occurred during the trial while one RCT indicated that no adverse events were recorded for the duration of their trial. ${ }^{19-22}$ All three studies reporting adverse events compared different doses of Fluzone vaccine including $3 \mu \mathrm{g}, 6 \mu \mathrm{g}, 7.5 \mu \mathrm{g}, 9 \mu \mathrm{g}$ and $15 \mu \mathrm{g}$ per strain doses.

Two of the studies were carried out in healthy adult populations and one RCT was conducted in older healthy adults ( $>60$ years of age) ${ }^{20-22}$ One RCT found that joint or muscle pain following vaccination was statistically significantly higher in the full dose $(15 \mu \mathrm{g})$ group compared with the half-dose $(7.5 \mu \mathrm{g})$ group and that while injection site pain initially appeared to be statistically significantly 


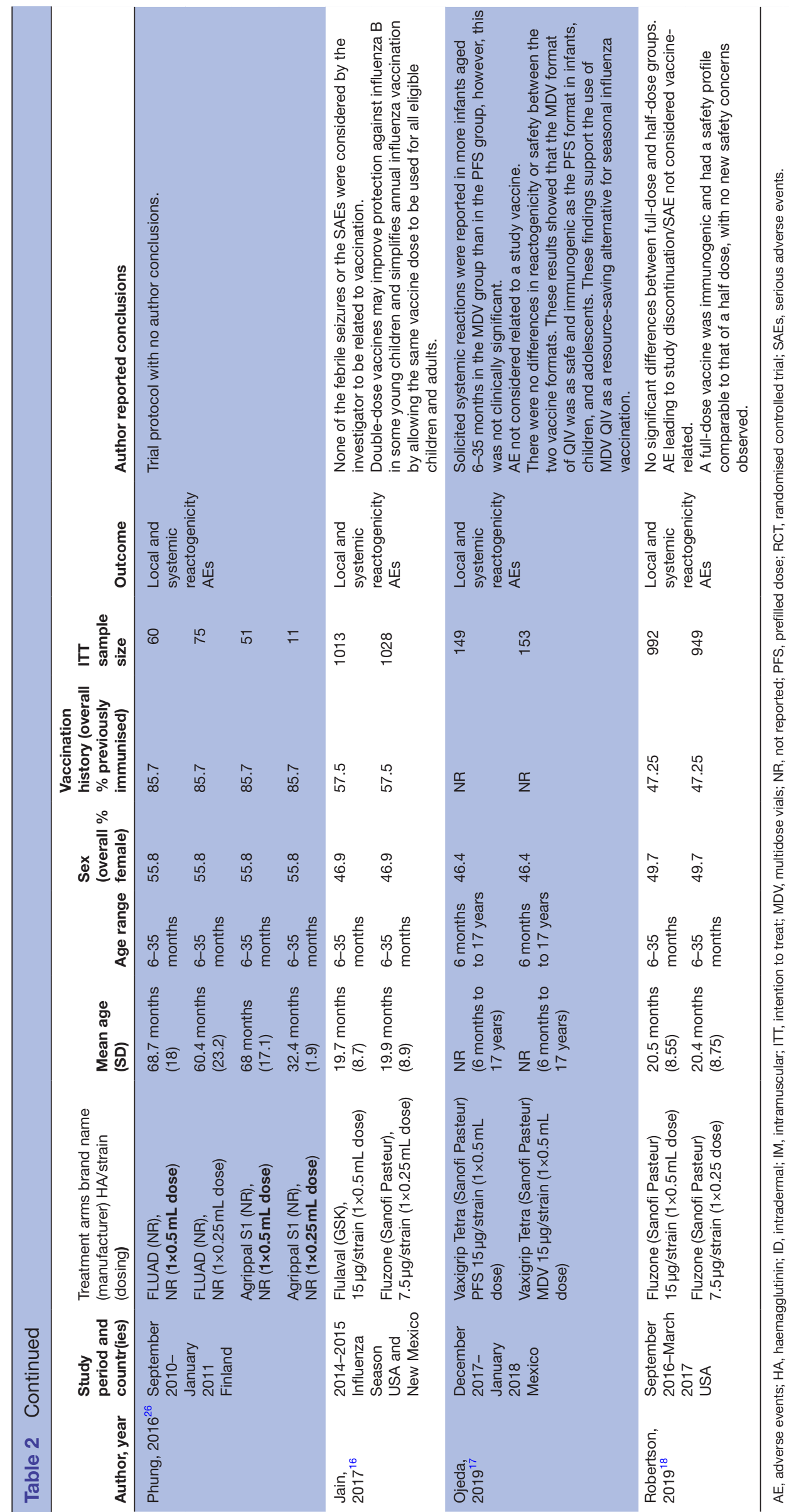




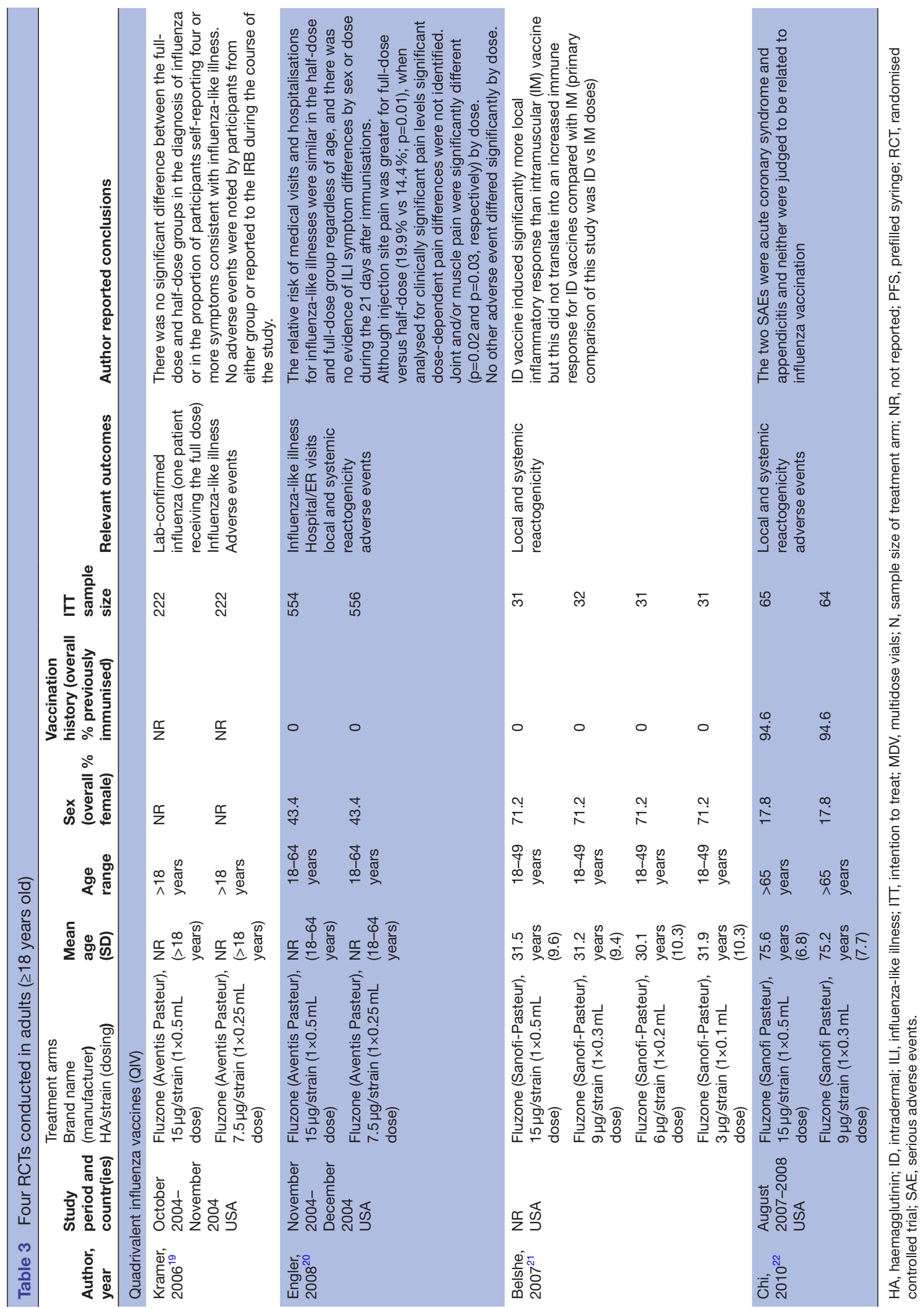


higher in the full dose group, when adjusted to include only clinically significant pain levels ( $>3$ out of 5 on a Visual Analogue Scale) the difference was no longer statistically significant. ${ }^{20}$ The RCT found no differences in occurrence or severity of any other adverse effects. Similarly, one RCT comparing four different doses of Fluzone (3 $\mu \mathrm{g}, 6 \mu \mathrm{g}, 9 \mu \mathrm{g}$, and $15 \mu \mathrm{g}$ per strain) did not report any differences between the IM vaccination groups. ${ }^{21}$ Finally, the RCT in older adults also found no difference in the occurrence or severity of adverse events in the low-dose $(9 \mu \mathrm{g})$ vs high-dose $(15 \mu \mathrm{g})$ group and found no serious adverse events that were considered related to the vaccine. $^{22}$

\section{DISCUSSION}

PHAC commissioned this rapid scoping review to identify the evidence for efficacy and safety of fractional influenza vaccine dosing for IM administration of seasonal influenza vaccines in healthy individuals of all ages that have been evaluated in human trials. Thirteen RCTs published between 2006 and 2019 comparing standard/full-dose and half/low-dose vaccines were included in this scoping review after a comprehensive search of three electronic databases, trial registries and references of relevant systematic reviews. The majority of the included RCTs were conducted in children and evaluated TIV.

In young, healthy children, there were no effectiveness outcomes of interest reported. However, local reactogenicity, systemic reactogenicity and adverse events were comparable across the full-dose and half-dose TIV and QIV vaccine arms. In addition, the authors of one RCT in children and adolescents that compared full-dose QIV using PFS vs MDV also found no statistically significant differences in safety outcomes between administration formats. In healthy adults (including older adults), halfdose QIV was considered equally effective as high-dose in the two RCTs that assessed clinical effectiveness. Safety profiles were similar across groups in all four RCTs.

A full systematic review with meta-analysis based on the studies and results of this scoping review was conducted by the NACI and the report was published in January of 2021. ${ }^{3}$ Briefly, the report found that there is some, but still insufficient, evidence that fractional doses of influenza vaccine provided via the IM route are effective and immunogenic in healthy individuals. NACI concludes that since many of those at high risk of influenza (eg, adults 65 years of age and older, individuals with specific underlying chronic health conditions) may have a lower immune response to influenza vaccination already (due to immunosenescence in older adults or a condition that alters immune function), it is important to ensure that those at high risk continue to receive the full dose of influenza vaccine. With regard to the safety of IM seasonal fractional doses of influenza vaccines, there is fair evidence that fractional doses do not result in significant differences compared with full dose with regard to severe adverse effects post-influenza vaccination. Readers are encouraged to reference the full NACI report on the Health Canada website. ${ }^{3}$

\section{Strengths and limitations}

A strength of this rapid scoping review was that it was conducted within a 6 -week timeline and the methods were tailored to provide results to the stakeholders within 4 weeks. We also did not restrict the search dates and study screening was completed independently by two reviewers. We developed a comprehensive search using three major databases, and searched the grey literature. We engaged with the NACI stakeholder group, who provided input on the PICO criteria, and funded this rapid scoping review.

We were limited by the lack of studies providing objective outcome data. Only one RCT by Kramer et al reported the objective outcome 'laboratory-confirmed influenza', and the other RCT by Engler only reported the outcome 'influenza like illness'. ${ }^{19} 20$ Since a 2014 narrative review found that less than $25 \%$ of cases diagnosed by physicians as influenza like illness were later laboratory proven influenza cases, ${ }^{23}$ we are lacking RCTs examining fractional dosing of IM influenza immunisation. Further, twelve dose-sparing RCTs were not included because they did not provide sufficient data, and did not include vaccines that were deemed of interest to the stakeholders. Another limitation was that only studies published in the English language were included, and data extraction was conducted by one abstractor and one verifier. Since this was a scoping review, we did not appraise the methodological quality of the included studies. ${ }^{24}$

\section{Future research}

Dose-sparing approaches such as ID immunisation vaccination exhibits similar, or even enhanced, immunogenicity, when using a fractional dose only, as compared with IM or subcutaneous immunisation, and should be explored in future scoping reviews. ${ }^{25}$

\section{CONCLUSIONS}

In our scoping review, we found 13 RCTs on the efficacy and safety of fractional doses of influenza vaccine provided via the IM route to healthy adults and children. These studies were used to inform a systematic review with meta-analysis which were commissioned by the PHAC. We found that due to the low number of studies in healthy adults, namely one study assessing laboratory confirmed influenza and two evaluating influenza-like illness in adults, there remains a need for further evaluation of the clinical effectiveness of IM dose-sparing strategies using vaccines currently available in this population.

Twitter Carole Lunny @carole_lunny

Acknowledgements The authors would like to thank Jessie McGowan for her assistance in developing literature searches, Tamara Radar for PRESS of literature search, Alissa Epworth for her assistance executing searches and retrieving articles, and Navjot Mann for her assistance in contacting author's and formatting this manuscript. 
Contributors CL wrote and revised the final manuscript. JA and PR screened citations and full-text articles, abstracted and verified data, interpreted results and wrote the first draft manuscript. CW and NR screened citations and full-text articles, abstracted data and reviewed the manuscript. SES and ACT developed the protocol, obtained funding, interpreted results and edited the manuscript.

Funding This work was supported through the Drug Safety and Effectiveness Network funded by the Canadian Institutes of Health Research, the funders had no involvement in the design, conduct or publication of this study. SES is funded by a Tier 1 Canada Research Chair in Knowledge Translation and the Mary Trimmer Chair in Geriatric Medicine; ACT is funded by a Tier 2 Canada Research Chair in Knowledge Synthesis.

Competing interests None declared.

Patient consent for publication Not applicable.

Provenance and peer review Not commissioned; externally peer reviewed.

Data availability statement All data relevant to the study are included in the article or uploaded as online supplemental information.

Supplemental material This content has been supplied by the author(s). It has not been vetted by BMJ Publishing Group Limited (BMJ) and may not have been peer-reviewed. Any opinions or recommendations discussed are solely those of the author(s) and are not endorsed by BMJ. BMJ disclaims all liability and responsibility arising from any reliance placed on the content. Where the content includes any translated material, BMJ does not warrant the accuracy and reliability of the translations (including but not limited to local regulations, clinical guidelines, terminology, drug names and drug dosages), and is not responsible for any error and/or omissions arising from translation and adaptation or otherwise.

Open access This is an open access article distributed in accordance with the Creative Commons Attribution Non Commercial (CC BY-NC 4.0) license, which permits others to distribute, remix, adapt, build upon this work non-commercially, and license their derivative works on different terms, provided the original work is properly cited, appropriate credit is given, any changes made indicated, and the use is non-commercial. See: http://creativecommons.org/licenses/by-nc/4.0/.

\section{ORCID iDs}

Carole Lunny http://orcid.org/0000-0002-7825-6765

Andrea C Tricco http://orcid.org/0000-0002-4114-8971

\section{REFERENCES}

1 PHAC. Seasonal influenza vaccination coverage in Canada, 20192020, 2020.

2 PHAC. Canadian immunization guide chapter on influenza and statement on seasonal influenza vaccine for 2019-2020, 2019.

3 PHAC. Recommendations on fractional influenza vaccine dosing, 2021. Available: https://www.canada.ca/en/public-health/services/ immunization/national-advisory-committee-on-immunization-naci/ recommendations-fractional-influenza-vaccine-dosing.html

4 Peters MDJ, Marnie C, Tricco AC, et al. Updated methodological guidance for the conduct of scoping reviews. JBI Evid Synth 2020;18:2119-26.

5 World Health Organization AfHPaSR. Rapid reviews to strengthen health policy and systems: a practical guide, 2017. Available: https:// www.who.int/alliance-hpsr/resources/publications/rapid-reviewguide/en/

6 Tricco AC, Lillie E, Zarin W, et al. PRISMA extension for scoping reviews (PRISMA-ScR): checklist and explanation. Ann Intern Med 2018;169:467-73.

7 Rubin LG, Levin MJ, Ljungman P, et al. 2013 IDSA clinical practice guideline for vaccination of the immunocompromised host. Clin Infect Dis 2014;58:e44-100.

8 Hervé C, Laupèze B, Del Giudice G, et al. The how's and what's of vaccine reactogenicity. NPJ Vaccines 2019;4:39.
9 Synthesi SR. Toronto, Canada: Knowledge Translation Program, St. Michael's Hospital 2012

10 Della Cioppa G, Vesikari T, Sokal E, et al. Trivalent and quadrivalent MF59(囚)-adjuvanted influenza vaccine in young children: a doseand schedule-finding study. Vaccine 2011;29:8696-704.

11 Skowronski DM, Hottes TS, Chong M, et al. Randomized controlled trial of dose response to influenza vaccine in children aged 6 to 23 months. Pediatrics 2011;128:e276-89.

12 Langley JM, Vanderkooi OG, Garfield HA, et al. Immunogenicity and safety of 2 dose levels of a Thimerosal-Free trivalent seasonal influenza vaccine in children aged 6-35 months: a randomized, controlled trial. J Pediatric Infect Dis Soc 2012;1:55-63.

13 Pavia-Ruz N, Angel Rodriguez Weber M, Lau Y-L, et al. A randomized controlled study to evaluate the immunogenicity of a trivalent inactivated seasonal influenza vaccine at two dosages in children 6 to 35 months of age. Hum Vaccin Immunother 2013;9:1978-88.

14 Halasa NB, Gerber MA, Berry AA, et al. Safety and immunogenicity of full-dose trivalent inactivated influenza vaccine (TIV) compared with half-dose TIV administered to children 6 through 35 months of age. J Pediatric Infect Dis Soc 2015;4:214-24.

15 A Phase IIIB, observer-blind, randomized, parallel groups, extension study to evaluate the immunogenicity and safety following a single intramuscular dose of FLUAD or Agrippal S1 influenza vaccines in healthy children previously vaccinated in the V70P5 study. [Internet], 2015. Available: https://www.clinicaltrialsregister.eu/ctr-search/trial/ 2010-021644-18/results [Accessed 14 Jul 2020].

16 Jain VK, Domachowske JB, Wang L, et al. Time to change dosing of inactivated quadrivalent influenza vaccine in young children: evidence from a phase III, randomized, controlled trial. J Pediatric Infect Dis Soc 2017;6:piw068.

17 Ojeda J, Arredondo JL, Salcedo P. Immunogenicity and safety of a multi-dose quadrivalent inactivated influenza vaccine in individuals aged 6 months to 17 years: a randomized phase III trial. Hum Vaccin Immunother. 2019:1-5.

18 Robertson CA, Mercer M, Selmani A, et al. Safety and immunogenicity of a full-dose, Split-virion, inactivated quadrivalent influenza vaccine in healthy children 6-35 months of age: a randomized controlled clinical trial. Pediatr Infect Dis $J$ 2019;38:323-8

19 Kramer JS, Durham C, Schroeder T, et al. Effectiveness of half-dose versus full-dose influenza vaccine in health care workers. Am J Health Syst Pharm 2006;63:2111-5.

20 Engler RJM, Nelson MR, Klote MM, et al. Half- vs full-dose trivalent inactivated influenza vaccine (2004-2005): age, dose, and sex effects on immune responses. Arch Intern Med 2008;168:2405-14.

21 Belshe RB, Newman FK, Wilkins K, et al. Comparative immunogenicity of trivalent influenza vaccine administered by intradermal or intramuscular route in healthy adults. Vaccine 2007;25:6755-63.

22 Chi R-C, Rock MT, Neuzil KM. Immunogenicity and safety of intradermal influenza vaccination in healthy older adults. Clin Infect Dis 2010;50:1331-8.

23 Thomas RE. Is influenza-like illness a useful concept and an appropriate test of influenza vaccine effectiveness? Vaccine 2014;32:2143-9.

24 Peters MD, Godfrey C, Mclnerney P. Chapter 11: Scoping reviews (2020 version). In: JBI manual for evidence synthesis. 2020. JBI, 2020.

25 Schnyder JL, De Pijper CA, Garcia Garrido HM, et al. Fractional dose of intradermal compared to intramuscular and subcutaneous vaccination - A systematic review and meta-analysis. Travel Med Infect Dis 2020;37:101868.

26 Phung A. Clinical trial results: A phase IIIB, observer-blind, randomized, parallel groups, extension study to evaluate the immunogenicity and safety following a single intramuscular dose of FLUAD or Agrippal S1 influenza vaccines in healthy children previously vaccinated in the V70P5 study [online], 2016. Available: https://www.clinicaltrialsregister.eu/ctr-search/trial/2010-021644-18/ results [Accessed 6 Jul 2020]. 